\title{
A REVIEW ON OPTIMIZATION OF DRUG DELIVERY SYSTEM WITH EXPERIMENTAL DESIGNS
}

\author{
NAZIA KHANAM ${ }^{* 1}$, MD IRSHAD ALAM², QUAZI MD AAMER IQBAL MD YUSUF ALI ${ }^{3}$, AQUIL-UR-RAHMAN \\ SIDDIQUI ${ }^{4}$
}

${ }^{1}$ Malla Reddy Institute of Pharmaceutical Sciences, Hyderabad, Telangana State, India, ${ }^{2}$ United States Pharmacopeia Ltd. Hyderabad, Telangana State, India, ${ }^{3}$ K. T. Patil College of Pharmacy, Osmanabad, Maharashtra, India, ${ }^{4}$ Shri Bhagwan College of Pharmacy, Aurangabad, Maharashtra, India

Email: nazia.khanam7@gmil.com

Received: 19 Dec 2017, Revised and Accepted: 13 Feb 2018

\begin{abstract}
The present review article aims at determining the various possible techniques available to enhance the quality, safety and efficacy of pharmaceutical formulations by exploring most suitable and practically applicable experimental designs and optimization techniques. As we know that pharmaceutical industries are constantly in search of novel ideas to improve quality by various optimization techniques, hence in present review article we shall discuss latest optimization techniques and experimental designs to achieve the best combination of product and process characteristics under the given set of conditions. Experimental designs and optimization techniques are the tools that are simultaneously and systematically used to identify various types of problems that may influence research, development and production of pharmaceutical formulations. These are organized an approach to determine the relationship between the factors affecting a process and the output of that process. The screening methods discussed here include factorial design, fractional factorial designs, full factorial design, mixture designs etc. Recently, different software has been used in implementing optimization techniques in pharmaceutical products to enhance product quality by using most suitable available facilities.
\end{abstract}

Keywords: Optimization Techniques, Quality by Design, Experimental Design, Factorial Design, Mixture Design, Taguchi Design

(C) 2018 The Authors. Published by Innovare Academic Sciences Pvt Ltd. This is an open access article under the CC BY license (http://creativecommons.org/licenses/by/4.0/) DOI: http://dx.doi.org/10.22159/ijap.2018v10i2.24482

\section{INTRODUCTION}

It is not always easy to get the solution of problems directly or instantly, so in those cases, we precede towards the various optimization methods in search of our answer. But as we know optimization is not as easy, as we have written, still it will lead to some conclusion [1]. The term optimization has been derived from optimize, that means to make as perfect, functional or effective as possible. In pharmacy, the word optimized was earlier used to suggest that a product has been improved to obtain the desired objectives of a development scientist, in pharmaceutical preparations or pharmaceutical processes; optimization is a technique of searching the best composition or experimental conditions. Optimization can be defined as the implementation of systemic approaches to obtain the best combination of product and/or processes characteristics under a given set of conditions or it can also be said as choosing the best element from some set of available alternatives [2].

The main aim of designing quality formulations is achieved by implementing various Optimization techniques (OT) like Experimental Design (ED), the terms Formulation by Design (FBD) and Quality by Design (QBD) indicates that quality in the product can be developed by using various techniques of ED [3-5]. Earlier, any new pharmaceutical formulation was designed by studying the influence of composition and process variables on dosage form characteristics, changing one single or separate factor at a time while keeping others constant, this technique was also called as changing one variable at a time or one factor at a time. By this technique, problem may be solved but it does not guarantee the true optimum concentration or process and the product obtained may be suboptimal. Some of the limitations of traditional methods are: unpredictable, uneconomical, timeconsuming, energy utilizing, unsuitable to plug errors, yielding only workable solutions, non-suitable to reveal interactions, energy utilizing etc. [2] So, in order to overcome these errors a novel approach was followed, which is optimization technique, that involves systematic ED [6]. The following steps represent the latest optimization techniques for drug delivery system in pharmaceuticals:

$>$ Defining objectives of the study and planning the experiment.

$>$ Screening of factors and factors influencing studies.
$>$ Response surface methodology using experimental designs.

$>$ Formulation and evaluation of drug delivery systems as per experimental design.

$>$ Computer-aided modelling and search for an optimum.

$>$ Validation of design of experiments methodology.

$>$ Scale-up and implementation in pharmaceutical production.

The objectives of optimization techniques are involved to maintain the quality, economy and safety of public and industry. Whereas the significance of optimizing a pharmaceutical product involved the discovery of important variables involved, cheaper and efficient way of formulating the product and improving the consistency and usefulness of quality specification in the formulation [7]. OT and ED are tools that are used specifically to examine various problems that are encountered during research, development and production. It is obvious that if experiments are performed randomly the result obtained will also be random. Therefore, it is essential to plan the experiments in such a way that the relevant information will be obtained.

Important terminology used in optimization

Variables

These are the measurements or values that are characteristics of the data. There are two types of variables, dependent and independent variables. Formulation and process variables are directly controlled by the formulator, these include ingredients. Dependent or secondary variables are the responses of in-progress material or the resulting drug delivery system; it is the result of independent variables.

\section{Factor}

The factor is an assigned variable like concentration, temperature, lubricating agent, drug-polymer ratio, polymer-polymer ratio etc. A factor can be qualitative or quantitative. A quantitative factor has a numerical value to it e. g., concentration ( $1 \%, 2 \%$ so on), a drug to polymer ratio (1:1, 1:2 etc). Qualitative factors are the factors, which are no numerical, e. g., Polymer grades, humidity condition, type of equipment etc, these are discrete in nature. The factor is Assigned 
and Independent variable that affects the product or output of the process. It is an assigned quantitative and qualitatively as follows:

Quantitative: A numerical factor is assigned to it. Example: Concentration- $1 \%, 2 \%, 3 \%$ etc.

Qualitative: These are non-numerical. Example-Polymer grade, humidity condition etc.

Level: The levels of a factor are values or designation assigned to the factor, e. g., concentration (factor) $1 \%$ will be one level, while $2 \%$ will be another level. Two different plasticizers are levels of grade factor. Usually, levels are indicated as low, middle or high level. Normally for ease of calculation, the numeric and discrete levels are converted to-1 (low level) and +1 (high level). The general formula for this conversion is $X-$ the average of the two levels Level =Half the difference of levels Where ' $X$ ' is the numeric value.

Response: Response is mostly interpreted as the outcome of an experiment. It is the effect, which we are going to evaluate i.e., disintegration time, duration of buoyancy, thickness, etc. It is an outcome of the experiment.

Response surface: Response surface representing the relationship between the independent variables X1 and X2 and the dependent variable Y.

Run or trials: Experiments conducted according to the selected experimental designs.

Screening: To sort out something from.

Contour Plot: Geometric illustration of a response obtained by plotting one independent variable against another, while holding the magnitude of response and other variables as constant.

Interaction: It is also similar to the term effect, which gives the overall effect of two or more variables (factors) of a response. For example, the combined effect of lubricant (factor) and glidant (factor) on hardness (response) of a tablet. From the optimization, we can draw a conclusion about Effect of a factor on a response i.e., change in dissolution rate as the drug to polymer ratio changes. The relationship between various factors and response, those are a quantitative change of a response as we change the factors and its levels. The contribution effect i.e., whether two factors are contributing additively or antagonistically for a response, e. g., any relationship between the concentration of lubricant and glidant on the hardness of the tablet or flow property of the granules. It gives the overall effect of two or more variables means lack of summation of factor effects.

Effect: The effect of a factor is the change in response caused by varying the levels of the factor. This describes the relationship between factors and levels.

Multiple Linear Regression Analysis: The technique which expresses mathematically in form of the quadratic equation the linear relationship between a various independent variable and the dependent variable (Response).

Orthogonality: When the effect is due to the main factor of interest and no interaction.

Confounding: Lack of Orthogonality is termed as confounding or aliasing.

Resolution: Measurement of the degree of confounding.

Effect: The effect of a factor is the change in response caused by varying the levels of the factor. This describes the relationship between factors and levels [8-10].

\section{Optimization parameters}

The optimization parameters are broadly divided into two types: Problem type and Variables [11].

\section{Problem type}

The problem type of parameters again grouped into:
- Constrained type: Constrained types are, restrictions placed on the system by means of physical limitations or perhaps by simply practical based. This can best explained by taking hardness of tablet and its disintegrating time in less than $15 \mathrm{~min}$.

- Unconstrained type: In unconstrained type, there are no restrictions placed on the system by means of physical limitations or perhaps by simply practical based. But in pharmaceuticals, there is always a limitation of a means of a physical limitation or perhaps by simply practically the formulator wishes to place or must place on a system.

\section{Variables}

There are several variables in pharmaceutical formulation and processing but generally, variables can be classified into:

- Independent variables: These are directly under the control of formulator. Example: mixing time.

- Dependent variables: these are not directly under the control of formulator. Example: Homogeneity of mixed granules.

\section{Experimental designs (ED)}

It is an approach where the process variables are first screened to determine which are important to the outcome (excipients type, percentage, disintegration time (DT) etc [12-14]. The second step is the 'optimization', when the best settings for the important variables are determined. It involves the use of 'mixture designs' for changing mixture composition and exploring how such changes will affect the properties of the mixture. Experimental design is a statistical design that prescribes or advises a set of combination of variables. The number and layout of these design points within the experimental region depends on the number of effects that must be estimated. Depending on the number of factors, their levels, possible interactions and order of the model, various experimental designs are chosen. Each experiment can be represented as a point within the experimental domain. The point being defined by its coordinate (the value is given to variables) in the space [8]. It is an organized method used to determine the relationship between the factors (Xs) affecting a process and the output of that process (Ys). Statistical Experimental Design (ED) refers to the process of planning the experiment in such a way that suitable data can be collected and analyzed statistically, resulting in a valid and objective conclusion. This approach is used to draw meaningful conclusions from experimental data [15-22].

\section{Advantages of ED}

> Better innovation due to the ability to improve processes.

$>$ Less batch failures.

> Greater regulator confidence of robust products.

$>$ More efficient technology transfer to manufacturing.

$>$ Replications of results are obtained.

\section{Uses of ED}

It is used to determine the causes of variation in the response, to determine the conditions under which the optimal (maximum or minimum) response is achieved, to compare responses at different levels of controlled variables and to develop a model for predicting response.

\section{Key steps for experimental design}

To obtain good results from ED the following steps are followed:

$>$ Set objective.

> Select process variables

$>$ Select an experimental design.

$>$ Execute the design.

$>$ Check that the data are consistent with the experimental assumptions. 
$>$ Analyze and interpret the results.

\section{Selection of ED}

The choice of an experimental design depends on the objectives of the experiments and the number of factors to be investigated.

\section{Objective of ED}

$>$ Comparative analysis.

$>$ Optimal fitting of regression model estimation.

$>$ Response surface method determination.

$>$ Optimizing response when factors are proportions of a mixture.

$>$ Screening.

\section{Types of ED}

There are various types of ED available out of which method we have to use depends upon the resources we have and the parameters we want to estimate.

\section{Factorial designs}

Factorial designs (FD) were initially used in the 19th century by John Bennet Lawes and Joseph Henry Gilbert. A factorial design allows the effect of several factors and even interactions between them to be determined with the same number of trials as are necessary to determine any one of the effects by itself with the same degree. Theses designs are very frequently used response surface designs. A factorial experiment is one in which all levels of a given factor are combined with all levels of every other factor in the experiment. These are generally based upon first-degree mathematical models. First, whenever we are interested in examining treatment variations, factorial designs should be strong candidates as the designs of choice. Second, factorial designs are efficient. Instead of conducting a series of independent studies we are effectively able to combine these studies into one. Finally, these are the only effective way to examine interaction effects. Latest application of FD includes optimization of ibuprofen fast dissolving tablets [23-25].

\section{Fractional factorial design}

Fractional factorial design is generally used for screening of factor. This design has low resolution due to less number of run. Although these designs are economical in terms of number of experiments, the ability to distinguish some of the factor effects is partly sacrificed by reduction in the number of experiments.

\section{Full factorial design (FFD)}

It is an experimental design, which uses dimensional factor space at the corner of the design space. Factorial designs (FD) are used in experiments where the effects of different factors or used in experiments where the effects of different factors or conditions on choice for simultaneous determination of the effect of several factors and their interactions. The simplest factorial design FD is the 2 factorial designs, where two factors are considered each at two levels, leads to four experiments, which are situated in 2dimensional factor space at the corners of a rectangle. If there are 3 factors, each at two levels, eight experiments are necessary which are situated at the corners of an orthogonal cube on a 3-dimensional space. The number of experiments is given by 2 , where ' $n$ ' is the number of factors. If the number of factors and levels are large, then the number of experiments needed to complete a factorial design is large. To reduce the number of experiments, fractional factorial design can be used (i.e., $1 / 2$ or $1 / 4$ of the original number of experiments with full factorial design). The fitting of an empirical polynomial equation to the experimental result facilitates the optimization procedure. The general polynomial equation is as follows: $\mathrm{Y}=\mathrm{B} 0+\mathrm{B} 1 \mathrm{X} 1+\mathrm{B} 2 \mathrm{X} 2+\mathrm{B} 3 \quad \mathrm{X} 3++\mathrm{B} 12 \mathrm{X} 1 \mathrm{X} 2+\mathrm{B} 13 \mathrm{X} 1 \mathrm{X} 3+$ $\mathrm{B} 23 \mathrm{X} 2 \mathrm{X} 3$--Where $\mathrm{Y}$ is the response, $\mathrm{X} 1, \mathrm{X} 2, \mathrm{X} 3$ are the levels (concentration) of the 1, 2, 3 factors and B1, B2, B3, B12, B13, B23, are the polynomial coefficients, B 0 is the intercept (which represents the response when the level of all factors is low). Olmesartan and carbamazepine formulations had been developed by using full factorial design $[7,26,27]$.

\section{Plackett-burman designs (Hadamard designs)}

Plackett-Burman designs (PBD) are special two-level FFDs used generally for the screening of factors. This design is generally used when we want to screen a high number of factors if we want to study the effect of 7 factors then we have to show four dummy factors. The interpretations of results in FFD, PBD and taguchi design (TD) are drawn with the help of Pareto chart and half normal plot. These designs prove to be effective screening designs when only main effects are of interest. They economically detect large main effects, assuming all interactions negligible when compared with few main effects. These designs are also called as saturated designs as it investigates $n * 4$ experiments i.e. $8,12,16,20$, etc that is suitable for studying up to $7,11,15,19$, etc factors respectively.

\section{Central composite design (Box-Wilson design)}

This design was developed by Box and Wilson. A better design that encompasses the advantages of factorial design or fractional factorial design or the star design, is the central composite design (CCD). It is composed of $+2 \mathrm{k}$ Factorial design or Fractional factorial design. For nonlinear responses requiring second-order models, central composite designs CCDs are the most frequently employed. A two-factor CCD is identical to a $32 \mathrm{FD}$ with the rectangular experimental domain at $\alpha= \pm 1$, On the other hand, the experimental domain is spherical in shape for $\alpha=$ $\sqrt{2}=1.414$. The CCD is quite popular in response surface optimization during pharmaceutical product development. Practical application of CCD is the formulation of glipizide [28].

\section{Box-behnken designs (BBD)}

A specially made design, BBD requires only three levels for each facto-l, 0 and +1 . It employing 15 experiments run with three factors at three levels. It is economical then CCD because $t$ requires less number of Trial. Implementation of BBD in tenofovir preparation and fabrication of nateglinide nanoparticles $[29,30]$.

\section{Taguchi design (TD)}

Taguchi refers to experimental design as "off-line quality control" because it is a method of ensuring good performance in the development of products or processes." It is also used for screening of factors and it provides 8 experimental runs for 7 factors. Example of TD is the preparation of glicalazide [31].

\section{Mixture design (MD)}

These designs represent the quantity of each substance present but on their proportions. The sum total of the proportions of all the excipients is unity, and none of the fractions can be negative. Therefore, the levels of different components can be varied with the restriction that the sum total should not exceed one. In pharmaceutical formulations with multiple excipients, the characteristics of the finished product usually do not depend on the quantity of each substance but depend on their proportions. Here, the sum total of the proportions of all excipients is unity and none of the fractions can be negative.

Therefore, the levels of the various components can be varied with the restriction that the sum total should not exceed one. In a twocomponent mixture, only 1 factor level can be independently varied, while in a three-component mixture, only two-factor levels can be independently varied. The remaining factor level is chosen to complete the sum to one. Hence, they have often been described as ED for the formulation optimization. For process optimization, designs like FD and CCD are preferred. There are several types of mixture designs, the most popular being the simplex designs. A simplex is the simplest possible $n$-sided fig. in a (n-1) dimensional space. It is represented as a straight line for two components, as a 2-D triangle for three components, as a 3-D tetrahedron for four components and so on. The design points are uniformly distributed over the factor space and form the lattice. The practical use of Mixture design (MD) can be observed in solubilization study of celecoxib [32].

\section{Screening designs (SD)}

These are used for detecting the important factor and their level which affect the quality of formulation, these designs generally support only the linear responses. 


\section{Response surface designs (RSD)}

These are used when we required exact image of response, estimating interaction and even quadratic effects. RSD generally support nonlinear and quadratic response and capable of detecting curvatures.

\section{Star design}

The star design is simply a 22 factorial design rotated over $45^{\circ}$ angle in the space. A center point is usually added, which may be replicated to estimate experimental error, so there will be three levels for each factor where the quadratic effect can be measured, but the interaction effect cannot be measured as in case of the FFD. In star design, $2 \mathrm{k}$ Factorial designs are rotated over $45^{\circ}$ in (k-i) direction in $\mathrm{k}$-dimensional space with a replicated center point. $\mathrm{K}$ is the number of factors in the design. This results in $2 \mathrm{k}+\mathrm{R}$ experiments, where $\mathrm{R}$ is the replicate of the center point.

\section{Box design (BD)}

In central composite design, each factor has five levels. If the number of factors increases, the number of experiments may become too high. $\mathrm{BD}$ is used when three or more factors are an economical alternative in which each factor is given three levels. The design is called an orthogonal balanced incomplete block design. It can be split into a set of incomplete blocks, which means that every effect is not estimated in every block, but every factor effect is measured as an equal number of times with a balanced partition over the different blocks.

\section{Doehiert hexagon or uniform shell design (USD)}

Doehiert proposed uniform shell designs (USD), starting with an equilateral triangle, mirrored in one side to a hexagon. The hexagon is expandable in 2-dimensional space by mirroring the center point in the outward sides. The equally spaced design points are uniformly distributed in concentric circles. It is also expandable in 3-dimension to concentric spherical shells. Due to the uniform distribution, models based on this design provide, a good basis for interpolation. A disadvantage may be that the number of levels is not same for all factors. The design may be started with one side of the hexagon parallel to the most important axis.

\section{Simplex lattice design (SLD)}

These designs are used to explore the interior and the boundaries of the simplex. The number of factors determines its dimensions. The pattern of design points in the factor space and their number depend on the degree (the term of the highest order) of the model that is postulated. The points are distributed orderly over the factor space, forming a lattice. The factors can be controlled accurately and precisely. The coefficients of model equation can be calculated easily.

\section{Extreme vertices design (EVD)}

It often occurs in formulation studies that the whole factor space is not accessible for experiment or that some areas are expected not to give useful responses. In an Extreme Vertices design, observations are made at the corners of the bounded design space, at the middle of the edges and at the center of the design space. These can be used for the mixture composition as well as in combination with factorial designs.

\section{D-optimal design (DOD)}

This design explains that these designs maximize the information in the selected set of experimental runs with respect to a stated model. The D-Optimal design (DOD) maximizes the determinant, of which is an overall measure of the information. Geometrically, this corresponds to maximizing the volume in a dimensional space. There is a linear constraint on the factor settings, reducing the experimental region to an irregular polyhedron. There are no classical designs that can well investigate an irregular region, then DOD is then the preferred choice as it makes efficient use of the entire experimental space. There are formulation factors, with lower and upper bounds, and possibly additional constraints, making the region an irregular polyhedron. There are qualitative factors, with more than two levels and there is no mixed level design available, or the mixed level design suggests too many runs to be acceptable. The objective is Response Surface Matter (RSM) and there are qualitative factors. The number of experimental runs affordable is smaller than the number of runs of any available classical design.

\section{Sequential optimization design (SOD)}

Despite numerous merits of simultaneous approaches, there are situations where there is hardly any prior knowledge about the effects of variables. Such situations call for the application of the sequential methods. In sequential approach, optimization is attempted in a step-wise fashion. Experimentation is started at an arbitrary point in the experimental domain and responses are evaluated. An important aspect of sequential designs is to know when the goal has been accomplished.

\section{Interpretation of experimental design data}

Assume that we have a final model that has passed all the relevant tests that includes visual and quantitative tests, then we and are ready to make conclusion and decisions. These should be the response to the outputs dictated by the original experimental goals [33-37].

\section{Optimization of important factors}

\section{Model development}

A model is an expression defining the quantitative dependence of a response variable on the independent variables. Usually, it is a set of polynomials of a given order or degree. From this polynomial equation, we calculate the coefficient with the help of principal of MLRA (Multiple Linear Regression Analysis). By the help of software, we can also study here the effect of excipients, their interaction study, 3D Response plot, Contour Plot etc. In screening design with the help of half normal plot and Pareto chart, we can find out easily the main factor and their level. From the models thus selected, optimization of one response or the simultaneous optimization of multiple responses needs to be optimized graphically, numerically and by using Brute force search technology.

$>$ Graphical Optimization (GO): It is also known as response surface analysis (RSA) deals with selecting the best possible formulation out of a feasible factor space region. To do this, the desirable limits of response variables are set, and the factor levels are screened accordingly by the help of overlay plot [38].

$>$ Brute-force search (Feasibility and Grid search): Brute-force search technique is the simple and exhaustive search optimization technique. It checks each and every single point in the function space. Herein, the formulations that can be prepared by almost every possible combination of independent factors and screened for their response variables. Subsequently, the acceptable limits are set for these responses, and an exhaustive search is again conducted by further narrowing down the feasible region. The optimized formulation is searched from the final feasible space that is called as grid search, which fulfils the maximum criteria set during experimentation.

$>$ Numerical Optimization: It deals with selecting the best possible formulation out of a suitable factor. To do this, the desirable limits of response variables are set, and the factor levels are displayed by the software. Other techniques used for optimizing multiple responses are canonical analysis, ANNs and mathematical optimization [39-41].

\section{Validation of model}

The predicted optimal formulation or the checkpoint is prepared as per optimum factor level and the responses evaluated. On comparison of results of observed and predicted response conclusion will be drawn for model validation.

The following points should be considered before selecting any software for Experimental Designs and Optimization techniques:

$>$ A simple graphic user interface (GUI) that's intuitive and easy-to-use.

$>$ A well-written manual with tutorials to get you off to a quick start.

$>$ A wide selection of designs for screening and optimizing processes or product formulations.

A spreadsheet flexible enough for data entry as well as dealing with missing data and changed factor levels. 
Graphic tools displaying the rotatable 3D response surfaces, 2D contour plots interaction plots and the plots revealing model diagnostics.

> Software that randomizes the order of experimental runs. Randomization is crucial because it ensures that "noisy" factors will spread randomly across all control factors.
Design evaluation tools that will reveal aliases and other potential pitfalls.

After-sales technical support, online help and training offered by manufacturing vendors.

Table 1: Various software used in optimization techniques

\begin{tabular}{ll}
\hline Software & Features \\
\hline Design Expert & Optimizing pharmaceutical formulations and processes; allows screening and study of influential variables for FD, FFD, BBD, CCD, \\
& $\begin{array}{l}\text { PBD and mixture designs; provides 3D plots that can be rotated to visualize the response surfaces and 2D contour maps; } \\
\text { numerical and graphical optimization }\end{array}$ \\
DE PRO XL and & MS-Excel compatible DE software for automated data analysis using Taguchi, FD, FFD and PBD. The relatively inexpensive \\
DE KISS & software, DoE KISS is, however, applicable only to the single response variable. \\
Mini Tab & Powerful DoE software for automated data analysis, graphic and help features, MS-Excel compatibility, includes almost all designs of RSM \\
MATREX & Excel compatible optimization software with facilities for various experimental designs and Taguchi design. \\
OPTIMA & Generates the experimental design, fits a mathematical equation to the data and graphically depicts response surfaces \\
OMEGA & Only for mixture designs; only program that supports multi-criterion decision making by Pareto-optimality, up to six objectives \\
& and has various statistical functions \\
FACTOP & Aids in the optimization of formulation using various FDs, and other designs through development of polynomials and grid \\
GRG2 & search; includes computer-aided-education module for optimization \\
& Mathematical optimization program to search for the maximum or minimum of a function with or without constraints [42].
\end{tabular}

\section{CONCLUSION}

After having a detailed study on the effect of OT and experimental designs ED in pharmaceutical formulations, we can conclude that these parameters are highly influential in enhancing the quality, safety and efficacy of pharmaceutical products. Optimization techniques are also helpfull in reducing the cost of the product by minimizing the number of experimental trials during formulation development. The optimized experimental designs are also effective in analyzing accurate quality assurances to regulatory authorities, regarding enhanced product quality. From the above study, it can be concluded that FD and CCD are most preferred designs for optimization. This field of study that is optimization of pharmaceutical formulations by various experimental designs ED is a recent trend of the investigation.

\section{ACKNOWLEDGEMENT}

All the authors are thankful to Principals of MRIPS Hyderabad, KTPCP Osmanabad and SBCP Aurangabad, for their support to carry out this work effectively.

\section{AUTHORS CONTRIBUTIONS}

All the authors have contributed equally.

\section{CONFLICT OF INTERESTS}

There is no conflict of interest from all the authors.

\section{REFERENCES}

1. Lachman L, Lieberman $H$. The theory and practice of industrial pharmacy. $3^{\text {rd }}$ ed. Varghese publishing house; 1990. p. 295.

2. Singh B, Bandyopadhyay S, Kapil R, Ahuja N. Systematic optimisation of drug delivery systems: an insight. Pharm Rev 2008;7:146-86.

3. Alam IM, Khanam N, Shaik JK, Ganguly S. Quality by design-a recent trend in pharmaceutical industries. World J Pharm Res 2016;5:608-20.

4. Singh B, Pahuja S, Kapil R, Ahuja N. Formulation development of oral controlled-release tablets of hydralazine: optimization of drug release and bioadhesive characteristics. Acta Pharm 2009;59:1-13.

5. Singh B, Gupta RK, Ahuja N. Computer-assisted optimization of pharmaceutical formulations and processes. Pharm Product Development 2006;2:746-895.

6. Singh B, Kumar R, Ahuja N. Optimizing Drug Delivery Systems Using Systematic Design of Experiments. PartI: Fundamental Aspects. Critical Reviews in Therapeutic Drug Carrier Systems February; 2005.

7. Shobha R, Hiremath R, Vanaja K. Optimization techniques in pharmaceutical formulation and processing. Textbook of
Industrial Pharmacy. Drug Delivery Systems, Cosmetic and Herbal Drug Technology; 2016. p. 158-68.

8. Kumar RG, Sanghvi I. Optimization techniques: an overview for formulation development. Asian J Pharm Res 2015;5:217-21.

9. Siddheshwar S, Varpe P, Waghmare G, Wadghule P, Vikhe D. Optimization techniques: a future of pharmaceutical product development. Int J Curr Trends Pharm Res 2014;2:491-3.

10. Schwartz J, Merck S, Dohme RL. Optimization techniques in product formulation. J Soc Cosmet Chem 1981;32:287-301.

11. Banker GS, Rhodes CT. Modern Pharmsceutics. $4^{\text {th }}$ ed. New York, Marcel Dekker, Inc; 2002.

12. Bolton S. Optimization techniques in pharmaceutical statistics. Practical and clinical Applications. $3^{\text {rd }}$ ed. New York: Marcel Dekker; 1997.

13. Anthony NA. Pharmaceutical experimental design and interpretation. $2^{\text {nd }}$ ed. Taylor and Francis Group; 2006.

14. Monica RP, Rao P. Preparation and evaluation of immediate release tablets of meroclopramide HCL using simplex centruoid mixture design. Int J Pharma Tech Res 2010;2:1105.

15. Lionberger RA, Lee SL, Lee L. Quality by design: concepts for ANDAs. Adv Appl Pharm Sci J 2008;10:268-76.

16. Nekkanti V, Muniyappan T, Karatgi P. Spray-drying process optimization for the manufacture of drug-cyclodextrin complex powder using the design of experiments. Drug Dev Ind Pharm 2009;35:9-29.

17. Patel MM, Amin AF. Design and optimization of the colontargeted system of theophylline for chronotherapy of nocturnal asthma. J Pharm Sci 2011;100:1760-72.

18. Robert AL, Sau LL. Quality by design concepts for ANDA. Adv Appl Pharm Sci J 2008;10:268-76.

19. Ajay SB. Evaluation of different composition of noisome to optimize Acelofenac transdermal delivery. Asian J Pharm Sci 2010;5:87-95.

20. Guidance for Industry. Immediate release solid oral dosage form. Center for Drug evaluation and Res; 1995. p. 1-22.

21. Noushine B, Naghmeh H. Formulation and optimization of captopril sublingual tablet using D-Optimal design. Iranian J Pharm Res 2008;7:259-67.

22. Patel MM, Amin AF. Design and optimization of the colontargeted system of theophylline for chronotherapy of nocturnal asthma. J Pharm Sci 2011;100:1760-72.

23. Lewis GA, Mathieu D. Pharmaceutical Experimental Design, New York, Marcel Dekker, Inc; 1999. p. 2, 80, 186, 487, 489.

24. Rani P, Veesam. Full factorial design in the formulation of lamotrigine suspension using locust bean gum. Int J Chem Sci 2013;11:751-60. 
25. Kumar SR, Yagnesh SNT, Kumar GV. Optimization of ibuprofen fast dissolving tablets employing starch xanthate using $2^{3}$ factorial design. Int J Appl Pharm 2017;9:51-9.

26. Vaddemukkala $Y$, Syed M, Srinivasarao. A research article on optimization of olmesartan tablet formulation by 23 factorial design. Int J Res Pharm Nano Sci 2015;4:188-95.

27. Barhatei S, Husain M. Development of hydrophilic matrix tablet of carbamazepine using $3^{3}$ full factorial experimental designs. Int J Pharm Sci 2015;7:369-75.

28. Dash RN, Habibuddin M, Touseef H, Ramesh D. Design, optimization and evaluation of glipizide solid self-nano emulsifying drug delivery for enhanced solubility and dissolution. Saudi Pharm J 2015;23:528-40.

29. Matlhola K, Seru LK, Tshweu L, Bahadur I, Makgatho G, Balogun M. Formulation and optimization of eudragit RS PO-tenofovir nanocarriers using box-behnken experimental design. Hindawi Publishing Corporation Journal of Nanomaterials 2015:11. http://dx.doi.org/10.1155/2015/630690

30. Gopi G, Kannan K. Formulation development and optimization of nateglinide loaded ethyl cellulose nanoparticles by the boxbehnken method. Int J Pharm Sci 2015;7:310-5.

31. Varshosaz J, Tavakoli N, Minayian M, Rahdari N. Applying the taguchi design for the optimized formulation of sustained release gliclazide chitosan beads: an in vitro/in vivo study. AAPS PharmSciTech 2009;101:9191-8.

32. Rahali Y, Wafaa E, Wartiti EM, Alaoui EY, Bouatia M, Laatiris A, et al. Solubilization of celecoxib using organic cosolvent and nonionic surfactants optimized by experimental design. Int J Pharm Sci 2016;8:161-6.
33. Khade MM, Sunil JP. Quality by design (QBD)-a quality improvement perspective for pharmaceutical development. Int J Pharm Res Bio Sci 2013;2:144-66.

34. Sumit K, Shikha T. A quantitative approach for pharmaceutical quality by design patterns. Invention Rapid Pharm Anal Quality Assurance 2012;4:1-8.

35. Naresh A. Formulation and evaluation of lansoprazole noisome. J Pharm Sci 2008;1:561-3.

36. Ranga S, Jaimini M, Sharma SK, Chauhan BS. A review on the design of experiments (DE). Int J Pharm Chem Sci 2014;3:216-24.

37. Hardik P, Shardda P, Bhavna P. A comprehensive review on quality by design $(\mathrm{QbD})$ in pharmaceuticals. Int J Pharm Sci Rev Res 2013;21:223-36.

38. Singh BM, Dahiya, Saharan V, Ahuja N. Optimizing drug delivery systems using "design of experiments. Part II: retrospect and prospects. Crit Rev Ther Drug Carrier Syst 2005;22:215-92.

39. Singh B, Ahuja N. Development of controlled release buccoadhesive hydrophilic matrices of diltiazem hydrochloride: optimization of bioadhesion, dissolution and diffusion parameters. Drug Dev Ind Pharm 2002;28:431-42.

40. Siddheshwar S, Varpe P, Waghmare G, Wadghule P, Vikhe D. Optimisation techniques: a future of pharmaceutical product development. Int J Curr Trends Pharm Res 2014;2:491-3.

41. Ladani R, Patel M, Patel R, Bhatt TV. Modern optimisation techniques in the field of pharmacy. Res J Pharm Bio Chem Sci 2010;1:148.

42. Reha S, Chodankar, Dev A. Optimisaton techniques: a futuristic approach for formulating and processing of pharmaceuticals. Ind J Pharm Bio Res 2016;4:32-40. 